\title{
El reformador J. L. Vives en la psicología y pedagogía alemana a comienzos del siglo XX
}

Strosetzki, Christoph

First published in:

“Una de las dos Españas...”, S. 845 - 858, Iberoamericana, Madrid 2007, ISBN 978-84-8489-292-2

Münstersches Informations- und Archivsystem multimedialer Inhalte (MIAMI) URN: urn:nbn:de:hbz:6-12429441350 


\title{
El reformador J. L. Vives en la psicología y pedagogía alemana a comienzos del siglo $\mathrm{XX}$
}

\author{
Christoph Strosetzki \\ (Westfälische Wilhelms-Universität Münster)
}

Puesto que el año de nacimiento de Vives fue 1492, en 1892 se presentó la ocasión de celebrar su $400 .^{\circ}$ aniversario. De hecho, y con motivo de este evento, se publicaron algunas traducciones alemanas de sus escritos. ${ }^{.}$Sin embargo, este hecho en sí no aclara el motivo por el que creció tanto el interés por Vives en torno al 1900 en Alemania, de tal manera que su obra pasó a ser punto central de numerosas tesis doctorales, así como artículos y libros científicos especialmente de filosofía, de pedagogía y de psicología. En todo caso, más allá del mero interés histórico, Vives parecía ofrecer respuestas al estado de discusión de las ciencias de aquel entonces. Posiblemente la situación alrededor de 1900 fuera de cambio paradigmático, comparable al que tuviera lugar de la Edad Media al Renacimiento.

A continuación se mostrará cómo fue la recepción de Vives en el contexto de la filosofía, particularmente de la teoría de la ciencia, antes de pasar a describir el valor que le fue asignado en el ámbito de la pedagogía y de la psicología. Con Harald Höffding se pone de manifiesto que cada una de las disciplinas, tanto en Vives como en la época de 1900, se veía en una relación de interdependencia con las demás. En 1907, en su manual de historia de la filosofía, Harald Höffding reconoce a Vives como «el precursor de la nueva psicología empírica» ${ }^{2}$ ya que éste pone la experiencia como base del conocimiento y emancipa la psicología de la metafísica y de la teología al no estar interesado en lo que es el alma sino en qué efectos tiene: Höffding, al igual que Vives, se muestra interesado en el análisis de los fenómenos del alma y sus funciones. Este punto de partida, común en ambos, cobra mayor importancia por cuanto que para Höffding el tratamiento de cuestiones psicológicas es imprescindible para resolver los demás problemas filosóficos. Por eso, en la introducción de su historia de la filosofía, trata en primer lugar la problemática psicológica coincidiendo así con una tendencia filosófica muy extendida en la época que ve la filosofía fusionada con la psicología. ${ }^{3}$

1 Wychgram 1883; Kayser 1896; Bröring 1897.

2 Höffding 1907: 7. Las citas alemanas se han traducido al español.

3 A este respecto se debe nombrar a Wilhelm Wundt. Éste entiende el mundo como desarrollo del espíritu, el cual él investiga con métodos experimentales de su psicología fisiológica. Franz Brentano y Theodor Lipps ven en la psicología la base de todas las ciencias filosóficas. Según Wilhelm Dilthey las ciencias humanas están basadas en la 
Es la psicología empírica de la que Vives cuenta como uno de sus fundadores. Es considerado seguidor de ese empirismo que rechaza un sistema científico derivado deductivamente. Así lo ve Wilhelm Windelband, el cual, en su obra titulada Geschichte der Philosophie, publicada en 1892, caracteriza a Vives como el enemigo de la hegemonía de los conceptos generales, en los que Vives ve la verdadera «razón de la depravación medieval de las ciencias». ${ }^{4}$ Vives, como empírico y crítico de la tradición y de la autoridad filosóficas, encuentra incluso un sitio de honor en la obra Geschichte des Materalismus de Friedrich Albert Lange. Éste compara a este autor renacentista con Leonardo da Vinci en el sentido de que ambos van más allá de la tradición de la Antigüedad y fundan «una ciencia de la experiencia independiente de Aristóteles y de toda la Antigüedad». ${ }^{5}$ De este modo, acentúa la gran importancia que tuvo Vives para su época como reformador de la filosofía y precursor de la crítica de Descartes.

La nada crítica aceptación de las doctrinas aristotélicas se enfrenta, por tanto, a la nueva ciencia de la experiencia asociada a la psicología de la que Vives cuenta como uno de los fundadores. En la octava edición de la obra de Friedrich Überweg Grundriss der Geschichte der Philosophie der Neuzeit que Max Heinze revisó y publicó en 1896, se presenta a Vives como antiaristotélico y reformador de la filosofía, puesto que, especialmente en el campo de la psicología, «aspiró a una ciencia de la experiencia que abandonara todas las tradiciones de la Antigüedad y, así, con este nuevo método defendió la investigación filosófica independiente». ${ }^{6}$ Especialmente en los campos de las ciencias naturales, la medicina y las matemáticas Vives exigió realizar una investigación independiente, la observación de los fenómenos y experimentar con ellos.

Theodor G. A. Kater presentó en una tesis doctoral del año 1908 la importancia de la crítica aristotélica que se le atribuía a Vives. Afirmó que el mérito principal de Vives estaba en la ordenación histórica de Aristóteles, cuya relevancia hay que situar en su tiempo y en el contexto de la historia de la filosofía, por lo que Aristóteles perdería su prestigio como autoridad atemporal. El logro de Vives en conjunto residiría más en la crítica que en los bosquejos positivos, es decir, en indicar los defectos y en sugerir cómo solventarlos. Asimismo, Kater afirma que la disciplina que para Vives representa punto de partida y fundamento no era la psicología, sino la pedagogía: «todos los hilos de su vida científica convergen en su pedagogía y constituyen un sistema muy bien pensado». ${ }^{7}$ Kater añadió otro punto de vista a este respec-

experiencia interior y en la comprensión de los otros y sólo en una psicología que analiza descriptivamente pueden encontrar un fundamento fiable. Véase Heinze 1896: 266 y ss., 274 y 279.

4 Windelband 1892: 284ss.

5 Lange 1974: 189.

6 Heinze 1896: 32.

7 Kater 1908: 3. 
to, o sea, la idea de una ciencia unitaria, tal y como en las primeras décadas del siglo XX postuló el círculo vienés, ${ }^{8}$ crítico de la filosofía especulativa y de la física. Como Vives, los miembros del círculo vienés tenían como objetivo la separación entre la metafísica especulativa y la ciencia empírica.

La crítica de Vives a la tradición parece, para Kater, ser en primera línea crítica a Aristóteles, al que se descalifica por su egoísmo, afán de disputa, engaño premeditado, ambición y su oscura manera de expresarse. El error fundamental de la física aristotélica - según Vives - reside en que generaliza demasiado rápidamente y que tiende a la especulación en extremo. La lógica de Aristóteles incluiría de modo inadmisible componentes metafísicos y gramáticos. Sin embargo, Kater corrigió y relativizó algunas de las críticas de Vives. Así, se muestra comprensivo con el conciso estilo aristotélico debido a que también otros pensadores «tienen que crear en cierto modo su propio idioma para tratar los enormes problemas con los que deben luchar». ${ }^{9}$ Por otra parte, la crítica que Vives practica a la dialéctica «arrọlló la lógica aristotélica e introdujo en su lugar los principios de la retórica y la gramáticà». En definitiva, Kater opina que en Vives no sólo tiene lugar un cambio paradigmático, sino también un giro de la crítica científica a la crítica lingüística. Este tema fue central también en la teoría de la ciencia del círculo vienés pocas décadas después del trabajo de Kater.

Vives no contemplaba las lenguas como finalidad en sí, sino como recurso. El conocimiento de muchas de ellas no significaba, para él, un rasgo de sabiduría. Vives, así como Comenius posteriormente, pensaba que el gran caos babélico de las lenguas era una desgracia y le hubiera gustado más disfrutar de la situación ideal de una lengua única, en la que las palabras expresaran perfectamente la naturaleza de las cosas. Comenius por su parte, así lo acentuó Nebe en 1891, persiguió - siguiendo la línea de Vives - la idea de construir una "muy significativa, muy real lengua» ${ }^{10}$ parecida a la protolengua que fuera más indicada para expresar la esencia de las cosas de lo que era el latín."

¿Se puede ensalzar a Vives por ser el fundador del empirismo moderno? A esta sugerencia se le opone al menos la tan extendida opinión de que este honor se lo tendría que llevar Francis Bacon por su obra Novum Organon (1620). En todo caso,

8 Véase en cuanto a la ciencia unitaria Kraft 1950: 5ss.; en lo que al análisis lógico de la lengua se refiere ver Kraft 1950: 12ss. y sobre el empirismo 77ss.

9 Kater 1908: 48.

10 Véase Nebe 1891: 29ss.

11 Vives no sólo fue apreciado como teórico de las ciencias sino también como humanista. Georg Eulitz pudo hacerse una idea de su habitación de estudios y de su círculo familiar a partir de la correspondencia mantenida durante veinte años entre Vives y Budé. Se ponen de manifiesto los esfuerzos de Vives por actuar de mediador entre Erasmo y Budé con motivo de sus desavenencias. Véase Eulitz 1897. Que en el campo de la pedagogía Vives se vio considerablemente influenciado por Erasmo lo demuestra Burger 1914. 
Rudolf Günther intentó en 1912 invalidar esta opinión por prejuiciada. Mostró paralelismos muy claros entre Vives y Bacon sacando así la conclusión de «que Bacon había conocido y leído la obra de Vives [...], así que nosotros con pleno derecho podemos considerar a éste como una fuente importante del autor inglés». ${ }^{12}$ Especialmente porque en general Bacon no indica sus fuentes, son las coincidencias más que simples casualidades: ambos critican las ciencias existentes, la preponderancia de la dialéctica, la hegemonía de Aristóteles, la inclusión en las reflexiones naturales de la causa final perteneciente a la teología, la sobrevaloración de los eruditos y la exagerada confianza puesta en la autoridad. Bacon opone la inducción al silogismo escolástico, a la conclusión deductiva. Tanto Bacon como Vives parten de la idea de que- todo conocimiento proviene de la percepción sensorial y de la experiencia. Bacon - àl igual que Vives - resulta ser un utilitarista puesto que sólo da importancia a la investigación de la verdad en cuanto que se pueda obtener de ella algún beneficio práctico.

La división, de orientación psicológica, de las ciencias según las tres partes del alma - memoria, imaginación y razón - en historia, poesía y filosofía no sólo se encuentra en Vives y en Francis Bacon sino que ya también en Roger Bacon (12191292). Además, Francis Bacon separa la teología natural de la teología revelada de un modo más claro que Vives: la primera sería junto con la filosofía natural y la antropología parte de la filosofía. En definitiva, Günther, después de numerosos análisis individuales, llegó a la conclusión de que había multitud de puntos «donde sólo Vives podría ser la fuente del autor inglés». ${ }^{13}$

La misma gran importancia que se le atribuyó a Bacon por ser el gran innovador de los métodos científicos, la tuvo Comenius en el ámbito de la pedagogía. Para J. L. Bohlen es «sin duda uno de los grandes pedagogos del siglo XVII». ${ }^{14}$ Así, tanto más relevante resulta la importancia del pedagogo Vives, bajo cuya influencia habrían estado - según Bohlen - no sólo Comenius, sino todos los pedagogos hasta la primera mitad del siglo XVIII. El mismo Comenius en la introducción a su Física ${ }^{15}$ declara que había recibido de Vives el primer impulso para ese trabajo. Además, pone de relevancia las exhortaciones de Vives acerca de la renovación de la filosofía y el modo de estudio y lamenta que Vives viera mejor lo que faltaba que cómo tendría que ser.

Asimismo, Alsted - que fue el profesor de Comenius en la reformada escuela superior de Herborn, perteneciente al ducado de Nassau - habría llamado la atención a su alumno sobre Vives, al que elogiaba con el siguiente juego de palabras: «Vives,

\footnotetext{
12 Günther 1912: 67.

13 Günther 1912: 65.

14 Bohlen 1906: 1.

15 Comenius 1633.
} 
qui vivet, quoad litterae vivent». ${ }^{16}$ August Nebe acentuó en 1891 cuán importante fue la influencia de Vives sobre el pedagogo Alsted. ${ }^{17}$ Éste, como Vives, parte de una profunda religiosidad, acentúa el principio de la utilidad en la educación y funda el uso de la didáctica y la pedagogía - frecuentemente utilizando la terminología de Vives - en la psicología, especialmente en la doctrina de la memoria. En todo ello rechaza la autoridad y la tradición en favor de la experiencia inductiva.

Las publicaciones de Paul Hause dirigidas contra Lange ${ }^{18}$ en 1890 acerca de la clase de influencia que Vives ejerció sobre Comenius preceden las de Nebe. En ellas Hause cita en primer lugar otras posibles fuentes, especialmente a Quintiliano, y el clima intelectual comparable en ambos autores. Posteriormente, a la lista con los doce paralelismos de Lange añade otros puntos y llega a la conclusión de que es probable «que Comenius, en sus opiniones y principios sobre la pedagogía, estuviera, si no directamente bajo la influencia de Vives, por lo menos sí estimulado». ${ }^{19}$

Lange citó los siguientes puntos en común entre los dos autores: para ambos la religión era el final absoluto de toda formación; ambos deseaban una religión unitaria de un bien entendido cristianismo; exigían que los métodos de enseñanza estuvieran en consonancia con la naturaleza, se debían descubrir los talentos naturales de los educandos, uno no podía darse por vencido ante estudiantes con poco talento, sólo se debía enseñar aquello que fuera de utilidad y que no perjudicara la religión o la moralidad, los profesores no podían ser gruñones, había también que tener en cuenta la lengua materna, los estudiantes tenían que adquirir interés por la materia de estudio sin ser forzados, había que introducir con cuidado los libros de autores paganos, en la enseñanza de cosas materiales se debía partir de las percepciones sensoriales y, finalmente, también la mujer necesitaba aprendizaje. A estos doce puntos comunes de Lange, añadió Hause otros aspectos en los que ambos autores también coincidían: las mujeres embarazadas tenían que prescindir de las comadronas, los pobres también debían ser aceptados en las escuelas, no había que descuidar el cuerpo ocupándose sólo del trabajo intelectual, tenían que animar a la compilación de sentencias, avisar de los malos amigos, acentuar la importancia de los modelos y de empezar cuanto antes con la instrucción y, finalmente, denunciar duramente las inconvenientes de su tiempo. En definitva resultan, por tanto, 21 puntos comunes.

Hause después de considerar el parecer de Vives con respecto a la educación de los niños hasta la edad en que son escolarizados, a la educación en lạ escuela, a las materias, al comportamiento de los niños, a los recreos, al cuidado corporal y a la diferente educación de niños y niñas, llega a la conclusión:

16 Véase Bohlen 1906: 13.

17 Nebe 1891.

18 Véase Lange 1887: 776-851.

19 Hause 1890: 68. 
Y así como todas las sugerencias pedagógicas de Vives estaban fundadas y además eran imprescindibles para aquel tiempo en el que el sistema educativo y escolar se encontraban en una situación muy precaria, así también se deben tomar en consideración en su mayor parte en nuestra época y en nuestra situación escolar. ${ }^{20}$

No obstante, en este pasaje se debe prescindir de la argumentación de Ruhmer sobre la concepción de Vives acerca de la mujer, pues parece:

como si Vives considerara la formación intelectual del género femenino mucho más necesaria que la del hombre ya que juzga el talento natural femenino de frívolo y principalmente propenso a las malas costumbres. ${ }^{21}$

Pero más importante es la cuestión acerca de qué obras pedagógicas de la Antigüedad tuvo en cuenta el humanista Vives. Que los escritos pedagógicos de Quintiliano fueron trascendentales para Vives, así como para Erasmo y otros humanistas lo probaron A. Messer en 1897 y J. M. Hofer en $1910 .{ }^{22}$ Hofer, al contrario que Messer, puso de relieve que Vives rechaza el ideal de la elocuencia de Quintiliano. Mientras que la mayoría de los humanistas apreciaban a Quintiliano como autor clásico de la pedagogía, el mérito de Vives consistiría - según el parecer de Hofer en haber roto con esta valoración de Quintiliano y, a pesar de los elementos comparables, haber construido «un nuevo sistema pedagógico fundamentalmente independiente del de Quintiliano». ${ }^{23}$ Puesto que Vives considera la historia del conocimiento como historia evolutiva, interpreta a Quintiliano, así como al anteriormente ya mencionado Aristóteles, como factor de relativa importancia en el desarrollo histórico. Desde el punto de vista cristiano Vives - a diferencia de Quintiliano - concede prioridad a otros aspectos como, por ejemplo, desterrar el discurso judicial del canon educativo por ser una controversia indecente, rechazar la ambición y validar la elocuencia formal sólo como medio y subordinada al contenido.

¿Cómo pudo Vives convertirse en uno de los puntos de referencia más importantes en la discusión pedagógica de comienzos del siglo XX? La pedagogía era en esta época una disciplina de moda, exactamente igual que la psicología; al menos, de ello evidencia August Baumeister, el editor de la obra Handbuch der Erziehungsund Unterrichtslehre für höhere Schulen. Él constató en 1894 en su folleto que las cuestiones pedagógicas se habían convertido en su época en el «niño mimado de la opinión pública»:

20 Hause 1890: 34.

21 Ruhmer 1915: 60. Asimismo tampoco pueden plantearse sus ideas calificadas de modernas por Weitzmann en 1905 acerca de la asistencia a los pobres en la obra De subventione pauperum, cuya referencia práctica reside en responder a la cuestión de cuáles son los cometidos que la necesidad de los pobres le plantean a la ciudad y a la administración municipal de manera que su cometido no siga siendo sólo un mero apoyo caritativo de los pobres y los necesitados sino que sea la eliminación de las enfermedades y la miseria. Würkert 1901: 8 y 17.

22 Messer 1897: 457-469; Hofer 1910.

23 Hofer 1910: 219. 
Cuestiones como las relativas al valor de las lenguas clásicas para la educación del presente o a la así denominada fatiga excesiva de los estudiantes preocupan a la prensa de todos los partidos; la rivalidad entre el instituto de bachillerato y la escuela secundaria se discute en asambleas y asociaciones públicas. ${ }^{24}$

En este contexto se le atribuye a Vives una gran importancia al ser valorado como modelo de reformador del sistema escolar:

Percibe la corrupción del mismo e investiga ante todo las causas de este desagradable estado tomando en consideración que primero se tiene que reconocer aquélla para encontrar remedio contra ella. ${ }^{2.5}$

Por ese mismo motivo Franz Kuypers, en su tesis doctoral de Kiel del año 1897 , tiene a Vives por el escritor pedagógico más significativo del siglo XVI y constata que

se concentra en él toda la reacción de la incipiente Edad Moderna contra los abusos pedagógicos de la baja Edad Media y que en él se une de modo parecido el germen de las reformas más significativas desde Sturm hasta Rousseau y se encuentran todas de nuevo fundidas en un todo. Él ha sido utilizado más veces de las que se han mencionado. ${ }^{26}$

Vives presentaría la variedad enciclopédica, la orientación hacia lo práctico, la crítica sana, la nominalización y realismo avanzados y la reconciliación del humanismo con la doctrina cristiana.

Para Vives es cometido de la pedagogía fomentar la pietas y la utilitas. En la educación de la voluntad y de la razón se tiene que tener en cuenta el-respectivo carácter individual. En relación con la memoria, Kuypers aprecia que Vives considere la posibilidad de que algo se puede dar por olvidado cuando todavía se encuentra en el inconsciente. Los factores más importantes de la educación que Kuypers observa en Vives son: la predisposición natural, la revelación cristiana, la familia y la escuela. Vives soluciona ese estado de decadencia de la ciencia y del sistema educativo, que él mismo constataría, por medio de la fundación de una academia ideal, cuya organización presenta minuciosamente conforme al tipo y estructuración de las asignaturas para las edades comprendidas entre los 7 y los 15 años, entre los 15 y los 25 años y a partir de los 25 años. Este tercer grupo se ocuparía de «la libre observación de la vida real». ${ }^{27}$ Aquí Kuypers acentuó que una estrecha familiaridad con la psicología era imprescindible porque - así argumenta siguiendo la tradición kantiana -: «nosotros no determinamos casi todo según la esencia de las cosas en sí, sino según las cualidades del intelecto». ${ }^{28}$

Si la teoría del conocimiento de Vives parte de la índole del conocimiento, es decir, de la psicología de la adquisición de conocimiento, entonces es natural que

24 Baumeister 1895.

25 Baumeister 1895: 105.

26 Kuypers 1897: 5.

27 Kuypers 1897: 65.

28 Ibíd. 
también la pedagogía dé importancia a la práctica de la adquisición de saber. Así se explica que Wilibald Kammel concediera a Vives un puesto honorífico en la historia de la didáctica experimental. ${ }^{29}$ Si bien Vives no conocía aún la observación estadística y experimental, sin embargo, podemos encontrar ya en él «el germen de la doctrina más relevante de la didáctica experimental moderna»; «en algunos detalles (asociación de ideas, estudio nocturno, cansancio, fluctuaciones de la energía) se muestra significativamente próximo a los caminos de la didáctica moderna». ${ }^{30}$ Para Vives el punto de partida es siempre la observación, ya sea de las propias experiencias o de los resultados de observación de sus precursores, de la cual, utilizando métodos inductivos, sabría derivar reglas de validez general. Las reglas, sin embargo, serían valiosas si tuvieran alguna utilidad práctica. La consideración del talento individual del estudiante juega un papel muy importante en la elección de una profesión y en la formación de la memoria.

De la premisa de tener en cuenta la individualidad se deriva la de incluir la propia intervención del estudiante en la adquisición de conocimiento. Paul Ilg. [sic] contemplaba en el año 1932 esta iniciativa propia como principio central en la pedagogía de Vives y el proceso educativo de éste lo veía condicionado tanto por un enriquecimiento de contenidos del intelecto como por la actualización de las disposiciones intelectuales. Puesto que para la transmisión del saber es preciso una iniciativa activa por parte del estudiante, habría que considerar especialmente la individualidad del estudiante. Vives coloca los diferentes tipos intelectuales en una lista, lo que le parecería especialmente moderno a Paul Ilg. ${ }^{31}$ Se debería distinguir entre tipos con una atención intensa y débil, fluctuante y fija; entre tipos con un modo de pensar sintético o analítico; entre tipos con talento para pensar productivo y reproductivo a finalmente entre tipos con una vida afectiva tranquila o excitable. Del principio de la individualización de Vives resulta - según Paul Ilg. - el del respecto a la edad e inteligencia del estudiante, en el que, conforme a la doctrina de los afectos, los afectos fundamentales de amor y odio se tienen que interpretar como congruencia o incongruencia. Vives, uniéndose aquí al concepto aristotélico de entelequia, entiende el proceso de aprendizaje como un desarrollo de talentos interiores que presupondría la voluntad y la disposición de aprendizaje y cierta apertura de mente.

Después de Vives, el postulado de tal fundamentación psicológica de la totalidad del trabajo educativo lo retomaría Pestalozzi con el mismo énfasis. El estudiante demostraría su iniciativa propia al observar, pensar y juzgar independientemente, con la propia lectura, al expresarse de modo autónomo y al practicar conocimientos y habilidades. Se trataría de despertar la actividad interior del estudiante y al potenciar este acto de involucrarse activamente, conducirle a una «formación de sí mis-

29 Kammel 1913: 36-45.

30. Kammel 1913: 45.

31 Ilg. 1931: 87. 
mo». En total, Paul Ilg. ve ya en Vives preformulado el principio metódico de Kerschensteiner, por el que la base de todo aprendizaje residiría en la propia iniciativa. ${ }^{32}$

Hasta aquí se ha demostrado que Vives fue apreciado en el marco de la historia de la pedagogía. Tampoco es menor el valor que se le atribuye en la historia de la psicología. W. Dilthey acentuó brevemente la importancia que supuso la concepción de los afectos de Vives para la historia de la psicología. ${ }^{33}$ En 1893 Roman Pade definió en un extenso estudio los afectos, a diferencia de los instintos, como emociones del alma dependientes del cuerpo, a las cuales precede «un reconocimiento y una valoración de lo bueno y lo malo del objeto». ${ }^{34}$ Los afectos se influirían recíprocamente:

Así se origina del amor hacia algo la esperanza de que ocurra, el deseo hacia ello, el miedo de que no ocurra, la alegría por la propiedad alcanzada, la tristeza por no conservarlo o por la pérdida. ${ }^{35}$

Pade presenta en detalle las ideas de Vives sobre las manifestaciones del amor, el egoísmo, y los demás afectos relacionados con el amor: simpatía, réspeto y compasión, alegría, esperanza, enojo, ira, odio, envidia, celos, indignación, tristeza, miedo, vergüenza y orgullo. La percepción de que Vives con su doctrina de los afectos había creado algo completamente nuevo, la corrige Pade, sin embargo, mostrando paralelismos con Tomás de Aquino y con la retórica aristotélica. De diferente manera que posteriormente el teórico Descartes, que con su doctrina dè los afectos presenta una psicología abstracta o Spinoza, el pensador matemático-apriorístico, el empírico Vives describe los afectos «como él los ha observado en el propio interior, en el frecuente contacto con personas de todas las clases sociales». ${ }^{36}$

Para probar que la psicología de Vives no pudo pasar sin las obras de la Antigüedad y de la Edad Media, Gerhard Hoppe presentó en 1901 en su tesis doctoral de Erlangen los dos primeros libros de De anima et vita. ${ }^{37}$ Él mostró ahí que se mencionaba frecuentemente a Aristóteles y en parte de manera crítica, que dominaban tanto. un componente platónico reforzado a través de estudios patrísticos como la anatomía y fisiología galénicas y que la escolástica estaba presente en segundo plano aunque Aristóteles apenas fuera mencionado.

La sistemática de Vives era, según Hoppe, ontológicamente amplia y diferenciaba en primer lugar entre un mundo inorgánico y otro orgánico. Este último tenía una vis interna, que condicionaría el crecimiento o la mengua. Después de las explicaciones sobre la alimentación, el crecimiento, la procreación y sobre los órganos

32 Ilg. 1931: 72. Véașe Kerschensteiner 1924.

33 Sobre la doctrina de los afectos de Vives, Dilthey 1969: 423- 429; véase también nota 3.

34 Pade 1893: 6.

35 Pade 1893: 8.

36 Pade 1893: 37.

37 Hoppe 1901. 
exteriores, la percepción interior se definía como la facultad de operar con objetos ausentes. Constaba de la functio imaginativa, que recogería las imágenes proporcionadas por los sentidos; la phantasia, que compondría de cada una de las percepciones sensoriales el objeto de los fenómenos y la facultas aestimatrix, que sopesaría instintivamente las ventajas e inconvenientes de las impresiones sensoriales. ${ }^{38}$ Para Vives el alma sólo sería accesible en una forma enturbiada por la materia, es decir, no en su esencia, sino en sus formas exteriores. Se encontraría "por todo el cuerpo». ${ }^{39}$ En el alma racional del hombre, que sería inmortal, se darían las siguientes diferencias: mens, o sea, intelligentia, voluntad y memoria. Cuando Vives parte de la percepción sensorial, cuya imagen la phantasia guarda en la memoria o induce la esencia del objeto con ayuda del entendimiento, le considera Hoppe un nominalista para èl que lo universal no existe en la realidad. ${ }^{40}$

Según Hoppe, Vives ve reforzada la memoria cuando en el momento de la aprehensión se añade un afecto. La asociación de ideas resulta cuando dos impresiones fueron percibidas por la fantasía al mismo tiempo y luego la aparición de una impresión tiene como consecuencia la aparición de la otra. En cuanto a la voluntad, Hoppe expone cómo Vives establece la relación con la acción, con el conocimiento, los afectos, la predestinación y la moral. En lo que concierne a la función aliviadora del sueño Hoppe encuentra también conocimientos en Vives:

Se les.debe, por tanto, conceder la posibilidad del sueño, a todos los seres vivos que estén dotados con el sentido interior de la fantasía. Cuando los hombres sueñan, junto con la fantasía actúa el entendimineto; sopesa, busca y encuentra frecuentemente en los sueños soluciones con las que se ha afanado despierto en vano. ${ }^{41}$

No obstante, Hoppe se refiere sólo a la doctrina de los temperamentos orgánicos (los humores), pero no al inconsciente.

En conjunto, Hoppe aprecia la «exigente demanda de un método empírico para la investigación psicológica y en relación con ello el rechazo por principio de un dogmatismo referente a las autoridades ${ }^{42}$ de Vives. A este respecto sería él, por tanto, el fundador de la psicología empírica aunque, por otra parte, en su argumentación, su propio postulado no le haga justicia y sí tome en gran parte la teoría de Aristóteles acerca de las primeras cualidades y de Galen la doctrina del pneuma y de los jugos. Lo mismo sirve para la consideración de Vives sobre la esencia del alma: apoyándose en Aristóteles aparece como effectio (energeia) del cuerpo y apoyándose en Platón se le adjudica una preexistencia. En los casos en los que Vives trabaja los pormenores - como en la doctrina de la asociación de ideas - le habrían salido bien

38 Hoppe 1901: 32-37.

39 Hoppe 1901: 45.

40 Hoppe 1901: 50.

41 Hoppe 1901: 92.

42 Hoppe 1901: 111. 
las observaciones por vía inductiva, donde «incluso la más nueva psicología no tendría que cambiar nada esencial». ${ }^{43} \mathrm{Si}$ bien Hoppe censura los a menudo inconsecuentes eclecticismos de Vives, alaba, sin embargo, que con Vives la psicología niega el servicio a la teología y empieza a constituirse como una disciplina independiente.

Esto no contradice la argumentación defendida por Georg Siske en su tesis doctoral $^{44}$ del año 1911. Según ésta, la psicología en Vives, en cuanto se pone en el contexto de la pedagogía, es relacionada inseparablemente con la ética y la teología. Mostró que Vives tiene en cuenta la filosofía y la ciencia primeramente según su importancia práctica como factor moralizante y no según su interés especulativo. El objetivo de la educación no sería la formación del intelecto sino que consistiría «en una educación de la voluntad que tomaría en consideración la vida entera de la psique humana, en harmoniosa formación del carácter religioso-moral». ${ }^{45}$

Puesto que, sin embargo, la autodeterminación también en Vives presupone conocimiento de uno mismo, éste no sería entonces el precursor de un nuevo voluntarismo extremo, como sostiene Fr. Paulsen, sino más bien el precursor de una posición que coordina voluntad y fuerza de conocimiento, en la línea de W. Wundt. De este modo, para Vives las leyes psíquicas tendrían mucha importancia como condiciones previas de las funciones de la voluntad y como bases para los principios de la formación de la voluntad y del carácter. Vives además posibilita percibir las normas pedagógicas y éticas desde una base psicológica segura.

Cuando Vives diferencia la percepción del mundo exterior, la çapacidad de imaginar objetos ausentes en la percepción interior y la capacidad de conocer objetos no sensoriales como los tres niveles de conocimiento, entonces esta teoría del conocimiento no estaría subordinada a la ratio speculativa, sino a la ratio practica.

También la cuestión de las ideas innatas, en Vives mantiene una orientación práctica en cuanto que el hombre en sus creaciones ha conservado una tendencia predominante a lo verdadero y una disposición para lo bueno. Siske considera digno de poner de relieve el intento de Vives de orientar la pedagogía éticamente puesto que esto se volvería a encontrar tras varios siglos en Schleiermacher y no se realizaría a fondo hasta Herbart. Así se demuestra que la ética de Vives es al mismo tiempo objeto de conocimiento y de fe. No necesitaría solamente ser obtenida por la vía de la revelación sino que sus principios básicos se podrían encontrar también por la vía del conocimiento filosófico. La formación de la voluntad y de la personalidad en Vives aparecen para Siske en un punto de intersección de pedagogía, ética, teoría del conocimiento, psicología y teología. Ve claro que, según Vives, las normas pedagógicas y éticas podrían ser percibidas empíricamente por medio de la observación psicológica. Ya que, aparte de eso, también se podrían derivar, o bien de la reflexión

43 Hoppe 1901: 117.

44 Siske 1911.

45 Siske 1911: 88. 
filosófica, o bien de la revelación religiosa, se muestra al mismo tiempo una división entre revelación, metafísica especulativa y ciencia empírica. De este modo, y a pesar de la interdependencia entre todas las disciplinas, la psicología no sería tampoco en Vives sierva de la teología.

Resumiendo se puede afirmar que las publicaciones en Alemania alrededor de 1900 subrayan de modo muy claro algunas convicciones de Vives. Una idea fundamental era que Vives emancipa la psicología frente a la metafísica y la teología ya que hace de la experiencia la base del conocimiento. Consecuentemente, no se pregunta por el ser del alma, sino por sus formas de expresión. Desde esta perspectiva se planteó también la cuestión filosófica del alma desde el punto de vista de la psicología. Ésta sería muy útil para tratar cuestiones filosóficas. Debido a que ya desde Kant no se planteó más conocer la esencia de las cosas en sí mismas, sino que se partió del modo de funcionamiento de la razón, la psicología apareció verdaderamente como disciplina fundamental. De esta disciplina sería Vives apreciado como pionero, el cual describiría los afectos como los habría observado empíricamente.

El empírico Vives fue considerado crítico de la tradición y de la autoridad filosófica. Tal y como Descartes, demostró ser antiaristotélico; relativizó a Aristóteles como factor importante en el desarrollo histórico y vio su fallo fundamental en tender a la generálización y la especulación, aunque Vives tomara de él algunos elementos. Mientras que Bacon, Comenius y Alsted se encuentran bajo la influencia de Vives, aparece Quintiliano - así como Aristóteles - como un factor de relativa importancia en el desarrollo histórico. Vives rechaza además su ideal de elocuencia por exagerado, ya que a Vives le parecen más importantes la variedad enciclopédica, la individualidad, la iniciativa individual, la orientación práctica, la crítica, el empirismo y el nominalismo como principios pedagógicos. Vives tematiza en su didáctica fenómenos observados empirícamente como, por ejemplo, la asociación de ideas, el cansancio o las fluctuaciones de energía. Además parece ambicionar la fundación psicológica de todo el trabajo educacional y así superar la mera deducción de las reglas de comportamiento de las normas éticas.

Un argumento para explicar el gran interés que se tenía por Vives en torno a 1900 es que la pedagogía y la psicología eran el centro de la atención científica y pública. A esto se añade que en él se veía el punto de partida de importantes tendencias contemporáneas, o sea, tendencias formuladas explícitamente en las primeras décadas del siglo XX, como la psicologización de la filosofía, la pedagogía como disciplina básica, la interdependencia de las disciplinas científicas, la ciencia unitaria y por último, y no menos importante, la teoría de la ciencia como crítica de la ciencia, cuya continuación en el siglo XX como crítica lingüística parecía ya apuntada en Vives. 


\section{Bibliografía}

Baumeister, August (1895): «Prospekt», en íd. (ed.): Handbuch der Erziehungs- und Unterrichtslehre für höhere Schulen. Tomo 1, $1^{\text {a }}$ parte. Theobald Ziegler: Geschichte der Pädagogik. München 1895.

Bohlen, Jann Lücken (1906): Die Abhängigkeit des Pädagogen Joh. Amos Comenius von seinen Vorgängern (tesis doctoral, Erlangen). Erlangen: Junge \& Sohn.

Bröring, J. (1897): Die Dialoge des J. L. Vives. Oldenburg i. Gr.

Burger, Otto (1914): Erasmus von Rotterdam und der Spanier Vives (tesis doctoral, Múnich). Kempten 1914.

Comenius Johann Amos (1633): Physicae ad lumen divinum reformatae Synopsis, Philodidacticorum et Theodidacticorum censurae exposita. Leipzig: Grossius.

Dilthey, Wilhelm ( $\left.{ }^{8} 1969\right)$ : «Die Funktion der Anthropologie in der Kultur des 16. und 17. Jahrhunderts», en íd.: Gesammelte Schriften. Stuttgart: Teubner / Göttingen: Vandenhoeck \& Ruprecht, tomo II, pp. 416-492.

Eulitz, Georg (1897): «Der Verkehr zwischen Vives und Budaeus», en: Jahresbericht des Königlichen Gymnasiums zu Chemnitz für das Schuljahr Ostern 1896 bis Ostern 1897. Edición de Bernhard Arnold. Chemnitz.

Günther, Rudolf (1912): Inwieweit hat Ludwig Vives die Ideen Bacons von Verulam vorbereitet? (tesis doctoral). Leipzig: Borna-Leipzig.

Hause, Paul (1890): Die Pädagogik des Spaniers Johannes Ludwig Vives und sein Einfluss auf Joh. Amos Comenius (tesis doctoral, Erlangen). Erlangen: Junge.

Heinze, Max (editor y revisor) (1896): Friedrich Überwegs Grundriss der Geschichte der Philosophie der Neuzeit. Berlin: Mittler.

Hofer, Johann Michael (1910), Die Stellung des Desiderius Erasmus und Jöh. Ludw. Vives zur Pädagogik des Quintilian (tesis doctoral, Erlangen). Erlangen.

Höffding, Harald (1907): Lehrbuch der Geschichte der neueren Philosophie. Leipzig: Reisland.

Hoppe, Gerhard (1901): Die Psychologie des Juan Vives nach den beiden ersten Büchern seiner Schrift «De anima et vita» dargestellt und beurteilt. Ein Beitrag zur Geschichte der Psychologie (tesis doctoral, Erlangen). Berlin: Mayer \& Müller.

Ilg., Paul (1931 [cubierta: 1932]): Die Selbsttätigkeit als Bildungsprinzip bei Joh. Ludwig Vives (1492-1540). Langensalza: Beyer (Friedrich Manns Pädagogischẹs Magazin).

Kammel, Wilibald (1913): «Johannes Ludovicus Vives und die experimentelle Didaktik», en: Pharus. Katholische Monatschrift fïr Orientierung in der gesamten Pädagogik. $4^{\mathrm{a}}$ promoción 1913, tomo 1, pp. 36-45.

Kater, Theodor Gustav Adolf (1908): Johann Ludwig Vives und seine Stellung zu Aristoteles (tesis doctoral). Erlangen.

Kayser, Friedrich (1896): Johannes Ludovicus Vives' Pädagogische Schriften. Einleitung, Charakteristik, Übersetzung und Erläuterungen. Freiburg im Breisgau: Herder.

Kerschensteiner, Georg $\left({ }^{2} 1924\right)$ : Das Grundaxiom des Bildungsprozesses, Leipzig.

Kraft, Victor (1950): Der Wiener Kreis. Der Ursprung des Neopositivismus. Ein Kapitel der jüngsten Philosophiegeschichte. Wien: Springer.

Kuypers, Franz (1897): Vives in seiner Pädagogik. Eine quellenmässige und systematische Darstellung (tesis doctoral, Kiel). Leipzig: Teubner.

Lange, A. (1887): «Vives», en Schmid, Karl A. (ed.), Encyklopädie des gesammten Erziehungs- und Unterrichtswesens. $2^{\mathrm{a}}$ edición. Leipzig: Fues, tomo IX, pp. 776-851. 
Lange, Friedrich Albert (1974 [ [21873]): Geschichte des Materialismus und Kritik seiner Bedeutung in der Gegenwart. Tomo 1. Ed. de Alfred Schmidt. Frankfurt am Main: Suhrkamp.

Messer, A. (1897): «Quintilian als Didaktiker und sein Einfluß auf die didaktischpädagogische Theorie des Humanismus», en: Jahrbuch für Philosophie und Pädagogik, $2^{\mathrm{a}}$ parte. Leipzig, sobre Vives: pp. 457-469.

Nebe, August (1891): «Vives, Alsted, Comenius in ihrem Verhältnis zueinander», en: Gymnasium zu Elberfeld. Bericht über das Schuljahr 1890-1891. Elberfeld.

Pade, Roman (1893): Die Affektenlehre des Johannes Ludovicus Vives. Ein Beitrag zur Geschichte der Psychologie. Münster: Aschendorff.

Ruhmer, Wilhelm (1915) Pädagogische Theorien über Frauenbildung im Zeitalter der Renaissance (tesis doctoral, Bonn). Bonn: Ludwig.

Siske, Georg.(1911): Willens- und Charakterbildung bei Johann Ludwig Vives (1492-1540) (tesis doctoral, Breslau). Langensalza: Beyer.

Windelband, Wilhelm (1892): Geschichte der Philosophie. Freiburg im Breisgau: Mohr.

Würkert, Georg (1901): «Ludwig Vives' Schrift von der Armenpflege», en: Jahresbericht der Städtischen Realschule mit Progymnasium zu Pirna 628, pp. 26ss., numeradas independientemente de la 1 a la 19.

Wychgram, J. (1883): J. L. Vives' Ausgewählte Schriften, en: Pädagogische Klassiker, de G. A. Lindner. Tomo XIV. Wien / Leipzig: Pichler. 